EPJ Web of Conferences 66, 09020 (2014)

DOI: $10.1051 /$ epjconf/ 20146609020

(C) Owned by the authors, published by EDP Sciences, 2014

\title{
Beam Diagnostics for Measurements of Antiproton Annihilation Cross Sections at Ultra-low Energy
}

\author{
K. Todoroki ${ }^{1, a}$, M. Hori ${ }^{2,1}$, H. Aghai-Khozani ${ }^{2,3}$, D. Barna ${ }^{1,4}$, M. Corradini ${ }^{5,6}$, T. Kobayashi ${ }^{1}$, M. \\ Leali $^{5,6}$, E. Lodi-Rizzini ${ }^{5,6}$, V. Mascagna ${ }^{5,6}$, M. Prest ${ }^{7,8}$, A. Soter ${ }^{1}$, E. Vallazza ${ }^{9}$, L. Venturelli ${ }^{5,6}, N$. \\ Zurlo $^{5,6}$, and R. Hayano ${ }^{1}$ \\ ${ }^{1}$ Department of Physics, University of Tokyo, Hongo 7-3-1, Bunkyo-ku, Tokyo 113-0033, Japan \\ ${ }^{2}$ Max-Planck-Institut fur Quantenoptik, Hans-Kopfermann-Strasse 1, D-85748 Garching, Germany \\ ${ }^{3}$ Physics Department, CERN, 1211 Geneva 23, Switzerland \\ ${ }^{4}$ Wigner Institute for Particle and Nuclear Physics, H-1525 Budapest, Hungary \\ ${ }^{5}$ Dipartimento di Ingegneria dell'Informazione, Università di Brescia, 25133 Brescia, Italy \\ ${ }^{6}$ INFN, Gruppo Collegato di Brescia, 25133 Brescia, Italy \\ ${ }^{7}$ Università degli Studi dell'Insubria, Italy \\ ${ }^{8}$ INFN, Sezione di Milano Bicocca, I-20126 Milano, Italy \\ ${ }^{9}$ INFN, Sezione di Trieste, I-34127 Trieste, Italy
}

\begin{abstract}
The ASACUSA (Atomic Spectroscopy And Collisions Using Slow Antiprotons) collaboration of CERN is currently attempting to measure the antiproton-nucleus in-flight annihilation cross sections on thin target foils of $\mathrm{C}, \mathrm{Pd}$, and $\mathrm{Pt}$ at $130 \mathrm{keV}$ of kinetic energy. The low-energy antiprotons were supplied by the Antiproton Decelerator (AD) and a radio-frequency quadrupole decelerator. For this measurement, a beam profile monitor based on secondary electron emission was developed. Data from this monitor was used to ensure that antiprotons were precisely tuned to the position of an 80mm-diameter experimental target, by measuring the spatial profile of 200-ns-long beam pulses containing $10^{5}-10^{6}$ antiprotons with an active area of $40 \mathrm{~mm} \times 40 \mathrm{~mm}$ and a spatial resolution of $4 \mathrm{~mm}$. By using this monitor, we succeeded in finely tuning antiproton beams on the target, and observed some annihilation events originating from the target.
\end{abstract}

\section{Introduction}

Several groups have previously measured the cross sections of in-flight antiprotons annihilating on targets of various mass numbers at kinetic energies $\mathrm{E}>600 \mathrm{keV}$, by using the Low Energy Antiproton Ring (LEAR) at CERN [1-6]. The ASACUSA (Atomic Spectroscopy And Collisions Using Slow Antiprotons) collaboration of CERN measured the antiproton-nucleus annihilation cross sections with targets of Mylar, Ni, Sn, and $\mathrm{Pt}$ at $\mathrm{E}=5.3 \mathrm{MeV}[7,8]$. These experimental results were consistent with the prediction of a modified black-disk model, which implies that the antiproton is strongly absorbed near the nuclear surface, and that the real potential is weaker compared to the imaginary one [9]. We are currently attempting to extend the measurements down to $\mathrm{E}=130 \mathrm{keV}$, where no experimental data exists and a deviation from the black-disk model may be observed [10, 11].

\footnotetext{
ae-mail: todoroki@nucl.phys.s.u-tokyo.ac.jp
} 


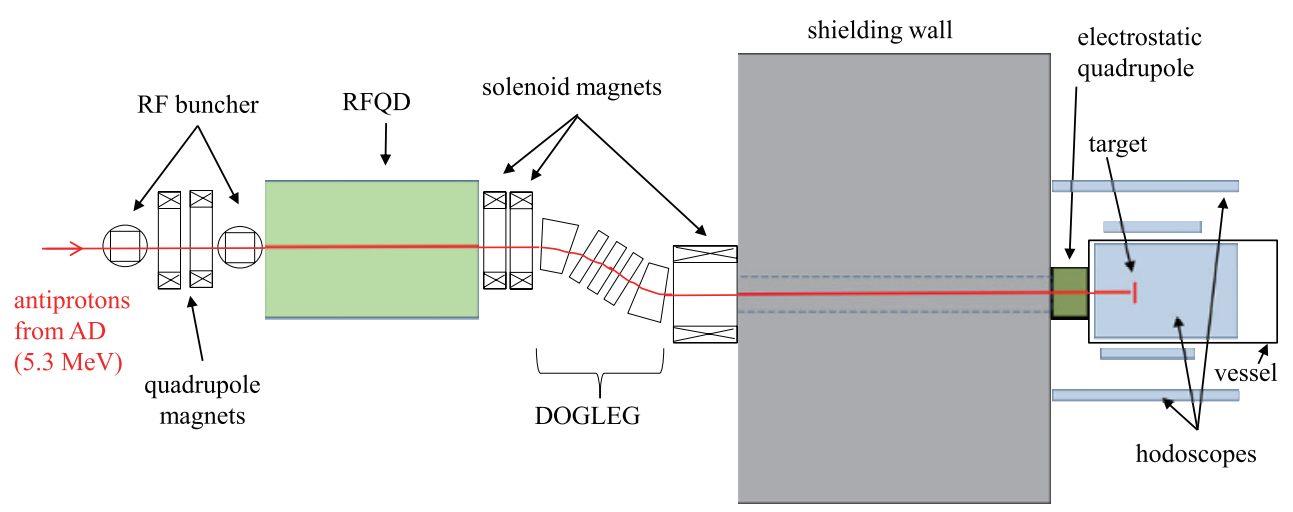

Figure 1. A schematic diagram of the ASACUSA beam line. This figure was taken from [10].

In this experiment, the 5.3-MeV antiprotons provided by the Antiproton Decelerator (AD) of CERN was allowed to pass through a radiofrequency quadrupole decelerator (RFQD), which reduced the kinetic energy of $30 \%$ of the antiprotons to $130 \mathrm{keV}$. An achromatic momentum separator was used to separate the decelerated antiprotons from ones which emerged from the RFQD with little or no deceleration. The 130-keV antiprotons were focused on 70-nm-thick target foils. The RFQD and momentum separator were originally developed to carry out laser spectroscopy of antiprotonic helium atoms $\left(\overline{\mathrm{p}} \mathrm{He}^{+}\right.$, see Figure 1) $[12,14,15]$.

As the AD provided a 200-ns-long pulsed beam containing $\sim 3 \times 10^{7}$ antiprotons, the instantaneous flux arriving at the experiment was too high to resolve individual annihilations. This made it difficult to distinguish the signals of annihilation events occurring on the target from backgrounds caused by spurious annihilations in the surrounding apparatus. A beam profile monitor was developed and placed at the target position. Data from this monitor was used to ensure that the antiprotons were precisely tuned to the 80 -mm-diameter experimental target. This monitor was a secondary electron emission detector with an active area of $40 \mathrm{~mm} \times 40 \mathrm{~mm}$ and a spatial resolution of $4 \mathrm{~mm}$.

\section{Beam Profile Monitor}

In this measurement, we used two kinds of profile monitors. The first of these was a nondestructive detector consisting of wire grids arranged in an XY configuration [16]. Each grid consisted of 32 gold coated tungsten wires of diameter $d=10 \mu \mathrm{m}$ stretched over a ceramic frame with a pitch $\Delta x=1 \mathrm{~mm}$ between neighboring wires. The detector had an active area of $32 \mathrm{~mm} \times 32 \mathrm{~mm}$. The photocathode grids biased at $-50 \mathrm{~V}$ were irradiated by the beam, and the secondary electrons emitted from them were accelerated toward the anode grids which were grounded. The beam profile was obtained by measuring the charge ejected from each of the 64 wires on the X- and Y-photocathode grids. Three monitors of this type were used in this experiment, placed at the entrance, middle, and exit of the momentum separator (see Figure 1).

The second detector was a beam profile monitor which was also based on secondary electron emission. It was used to measure destructively the spatial profile at the target position with an active 
area of $40 \mathrm{~mm} \times 40 \mathrm{~mm}$, and a spatial resolution of $4 \mathrm{~mm}$ [17]. The spatial profile of the antiproton beam was obtained by measuring the charge induced by antiprotons striking the segmented anodes and releasing electrons. The advantages of this detector included the simple design and low cost. This detector was required to be lightweight $(\sim 80 \mathrm{~g})$ so that it could be mounted on a delicate manipulation arm of the experimental target. The charge induced by antiprotons was transmitted by a 2-m-long ribbon cable with Kapton insulation and a vacuum feedthrough to the outside of the experimental apparatus. The signal was measured by hybrid charge-sensitive preamplifiers [18]. Figure 2 shows measured profiles of a pulsed beam containing $10^{5}-10^{6}$ antiprotons at the target position.

This detector consisted of anode pads fabricated on a four-layered circuit board of thickness $\mathrm{t}=$ $2 \mathrm{~mm}$ made of FR4-type glass epoxy. On the first layer (upstream side), there were 400 pads arranged in a checker-board pattern. Rows of 20 neighboring electrodes were electrically connected by a strip circuit pattern embedded in the second layer. Columns of 20 neighboring electrodes were similarly connected by a strip circuit pattern in the third layer. The fourth conductive layer provided electromagnetic shielding. In this way, the X-and Y-projections of the beam profile were measured. Electrical connections between the layers were established by through-hole vertical interconnect accesses (vias) of diameter $\mathrm{d} \sim 500 \mu \mathrm{m}$. These vias were filled by epoxy. The epoxy surfaces were then electrolytically plated with copper, nickel, and gold layers of respective thicknesses $\mathrm{t}=40 \mu \mathrm{m}, 1 \mu \mathrm{m}$, and $70 \mathrm{~nm}$. All other surfaces of the circuit board were similarly plated and grounded to avoid charge up, and minimize outgassing in vacuum. The insulators separating the anodes and cathodes were made of alumina of purity $99.5 \%$. Generally, ceramic boards [16] would provide a better ultra-high vacuum compatibility due to their lower outgassing properties, but we decided to use glass epoxy due to the modest vacuum requirement (in this measurement, it was $10^{-7} \mathrm{mbar}$ ) and light weight, as well as its lower cost and faster delivery.

The signal produced by the antiproton beam was processed in the following way. The charge induced on each strip electrode was transmitted to the outside of the experimental apparatus, using a 2-m-long ribbon cable and vacuum feedthrough. The signal then entered a hybrid charge-sensitive

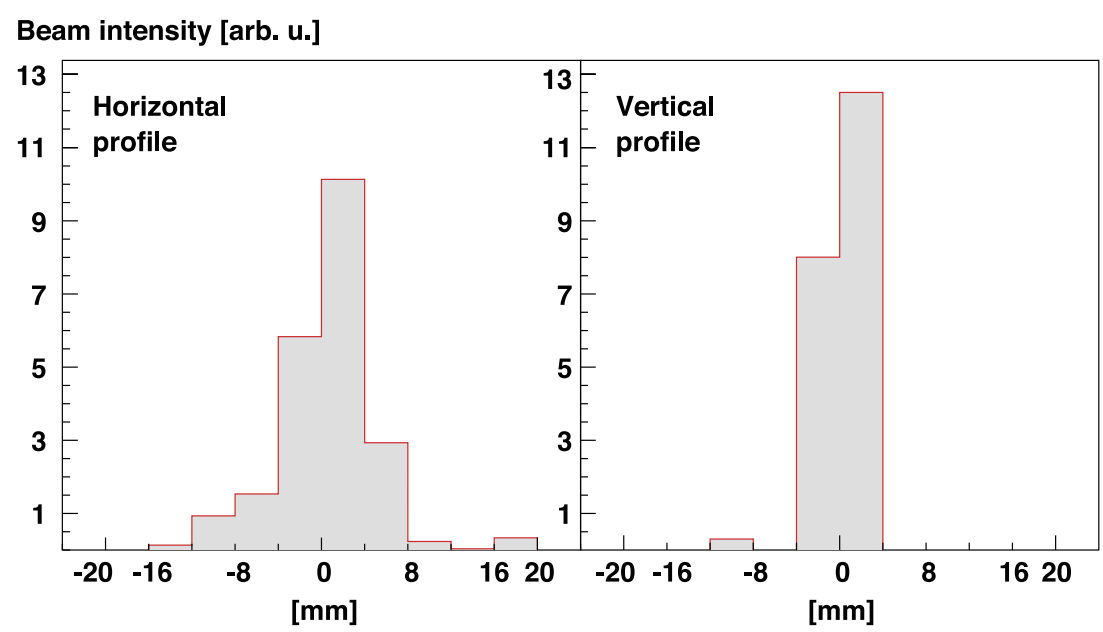

Figure 2. A spatial profile of the antiproton beam measured at the target position. This figure was taken from [10]. 
preamplifier through a decoupling capacitor $\mathrm{C}_{\mathrm{d}}=6.8 \mathrm{nF}$ [18]. This preamplifier had a feedback capacitor and resistor of $\mathrm{C}_{\mathrm{f}}=1 \mathrm{pF}$ and $\mathrm{R}_{\mathrm{f}}=1000 \mathrm{M} \Omega$ with a charge-to-voltage conversion ratio of $\sim 1 \mathrm{~V} / \mathrm{pC}$. This signal was transmitted to an active filter amplifier housed in an electronic rack over several meters of a twisted-pair cable to avoid picking up any external electromagnetic interference. The active filter amplifier consisted of a differentiator and four integrators. This amplifier shaped the signal into a Gaussian pulse with a peak time constant $\tau_{\mathrm{p}}=2 \mu \mathrm{s}$. The amplitude of this signal was measured by a peak-sensing analog-to-digital converter.

\section{Conclusion}

Despite the simplicity and low cost of this monitor, it had adequate sensitivity to measure the profiles of beams containing $\sim 10^{4}$ antiprotons. We succeeded in finely tuning a pulsed antiproton beam using this monitor so that the measurement of antiproton annihilation cross sections could be carried out (see Figure 2). The experiment was performed in 2012, and we observed some annihilation events originating from the targets [10]. Data taken in the experiment is now being analyzed to try to determine the cross sections.

\section{Acknowledgements}

The authors are grateful to ASACUSA collaborators and M. Mitani. The development of the profile monitor was supported by the Grant-in-Aid for Specially Promoted Research (20002003) of MEXT, Japan, the European Science Foundation (EURYI), and the European Research Council (ERC-Stg).

\section{References}

[1] A. Bertin et al., Phys. Lett. B 369, 77 (1996).

[2] A. Zenoni et al., Phys. Lett. B 461, 405 (1999).

[3] A. Zenoni et al., Phys. Lett. B 461, 413 (1999).

[4] W. Brückner et al., Z. Phys. A 335, 217 (1990).

[5] A. Bianconi et al., Phys. Lett. B 481, 194 (2000).

[6] A. Bianconi et al., Phys. Lett. B 492, 254 (2000).

[7] A. Bianconi et al., Phys. Lett. B 704, 461 (2011).

[8] M. Corradini et al., Nucl. Instr. and Meth. A 711, 12 (2013).

[9] J. Lichtenstadt et al., Phys. Rev. C 32, 1096 (1985).

[10] H. Aghai-Khozani et al., Eur. Phys. Journ. Plus 127, 125 (2012).

[11] C. J. Batty, E. Friedman, and A. Gal, Nucl. Phys. A 689, 721 (2001).

[12] M. Hori et al., Phys. Rev. Lett. 91, 123401 (2003).

[13] Y. Bylinsky et al., Proc. of 20th International Linear Accelerator Conf. 554 (2000).

[14] M. Hori et al., Phys. Rev. Lett. 96, 243401 (2006).

[15] M. Hori et al., Nature 475, 484 (2011).

[16] M. Hori, Rev. Sci. Instr. 76, 113303 (2005).

[17] K. Todoroki and M. Hori, JINST 7, C02052 (2012).

[18] M. Hori, Nucl. Instr. and Meth. A 522, 420 (2004). 